\title{
Mobile and wearable computing in patagonian wilderness
}

\author{
Samuel Almonacid (凶) $)^{1,2}$, María R. Klagges ${ }^{3}$, Pablo Navarro ${ }^{1,4}$, Leonardo \\ Morales $^{1,4}$, Bruno Pazos ${ }^{1,4}$, Alexandra Contreras Puigbó ${ }^{5}$, and Diego \\ Firmenich ${ }^{1}$ \\ 1 Departamento de Informática (DIT), Facultad de Ingeniería, Universidad Nacional \\ de la Patagonia San Juan Bosco, Trelew, Chubut, Argentina, \\ informaticatw@ing. unp.edu.ar, \\ WWW home page: http://http://www.dit.ing.unp.edu.ar \\ 2 Centro Para el Estudio de Sistemas Marinos (CESIMAR), Centro Nacional \\ Patagónico (CENPAT - CONICET), Puerto Madryn, Provincia de Chubut, Argentina \\ ${ }^{3}$ Instituto de Diversidad y Evolución Austral (IDEAus), Centro Nacional Patagónico \\ (CENPAT - CONICET), Puerto Madryn, Provincia de Chubut, Argentina \\ 4 Instituto Patagónico de Ciencias Sociales y Humanas (IPCSH), Centro Nacional \\ Patagónico (CENPAT - CONICET), Puerto Madryn, Provincia de Chubut, Argentina \\ 5 Departamento de Biología, Universidad Autónoma de Santo Domingo, Santo \\ Domingo, DN, República Dominicana
}

\begin{abstract}
Recent advances in mobile and wearable technology in the last few years have made the optimization of data collection processes possible in diverse fields.

Users currently have access to small portable devices that are not only sensitive to their activity, but also to their interaction with their environment.

These growing technological advances are in constant development, and have given way to the study and redesign of processes that can be tailored to fit any particular needs. Even users that are far from urbanization, without access to electricity can make use of these possibilities. These technologies can substantially improve their productivity, by allowing them to concentrate solely on their own tasks instead of on the interactions with the computational method used to support their activities. This study presents results and indicators relating to the application these tools within the field of Flora information retrieval, in areas far from urban centers.
\end{abstract}

Keywords: Mobile Computing, Wearable Computing, Context-Aware Computing

\section{Introduction}

The smart mobile device user population has grown boundlessly in the last few years. Globally, not just in Argentina, the current availability and widespread access to these devices serves to forecasts the probability that in a few years 
(halfway through the next decade), every digitally active person will have these devices or likely even more powerful computational environments (see [1-3]).

Moreover, the way users interact with this technology evolves so fast that in most cases it is impossible to predict the environmental and social impacts it may cause.

The ubiquity levels reached by the scientific and the information industry in their applications have allowed a massive expansion in terms of what a common user can virtually do with their own devices. A new demand for software products that are ready-made for using these capabilities can be seen as a possible result of this evolution.

On the other hand, given that the methods, techniques, and technologies involved in the development of these kinds of products have been in constant growth for the last few years, there is a wide range of possibilities relative to the development of platforms. Being completely up to date in every project can sometimes be a challenging task for professionals and students within this area of specialization.

In this paper, we present not only the results of applying this technology to a highly versatile domain, but also the benefits obtained from implementing this technology to the flora surveying methods tasks done by botanologists in our community.

Results were obtained after four years of research, with designs and development in mobile and wearable software ([4]). The impacts of applying this technology to the subject matter was then measured through a series of controlled experiments performed by an interdisciplinary biologist and programmer team.

Due to the encouraging results obtained from the experiments, these technologies were then incorporated by the biologists for their use in real case scenarios, and this allowed us to compare real results with the experimental results obtained from the previous phases.

The next chapter describes the domain of the aforementioned application, in order to facilitate an in depth comprehension of further sections. Chapter three describes the application developed, while chapter four summarizes the experimental results obtained with these applications. Finally, chapter five compares the experimental results with the values obtained from their use in real case uses. Conclusions and future works can be found in the last section of this document.

\section{Application Domain}

In terms of what the application domain is, this one in particular is based on vegetation surveys. While many different methods of surveying, measurement and ecological analysis exist, the need to rely on practical information is very important in order to obtain results that match up with reality as precisely as possible $([5])$. An adaptation of the "Point Quadrant" method (detailed in [6]) was used to adequately study the patagonian pasturelands, because this is a non destructive objective technique that easily allows for the evaluation 
of the different plant communities. This method is based on registering along a horizontal transect line, divided in 100 equidistant points, every single time plant species come into contact with a needle's tip as it is being let down in each $\operatorname{point}([6,7])$.

It should be noted that this method was implemented in remote areas far removed from urbanization, where the possibility of accessing an internet connection or even electricity is null. Biologists carry out three to six day surveying campaigns. During these campaigns they travel to the different points of interest within the area in one or more vehicles and perform an intensive supervised data collection on the area. In each transect at least 100 elements are registered concerning the species of flora found. The distance between the points of each transect depends on the estimated vegetation coverage. In areas where the coverage is greater than $65 \%$, it is recomended to record each step, between $45 \%$ y $65 \%$ every two steps and less than $45 \%$ every three steps, the latter being the most common ([7]). The first point would be located at $200 \mathrm{~m}$ from the roads and fencing in order to lessen the border effect produced by fragmentation ([8]). Besides the location of the transect, information pertaining the state of soil, the species, notes and pictures are also registered. Each person in charge of carrying out the survey, in deviating $200 \mathrm{~m}$ from the established pathways, must also walk at least the complete length of the $300 \mathrm{~m}$ transect and return to the vehicle; in doing so, they would have traversed approximately $800 \mathrm{~m}$ in total for every survey $(500 \mathrm{~m}$ from the first point to the last point of the transect and an additional $300 \mathrm{~m}$ to walk back to camp, the latter only if a shorter route back was available).

During campaigns the survey work starts at dawn and ends at sundown in order to maximize the amount of work that can be done under natural lighting, which is why the number of transects that can be successfully completed in a single day varies depending on the season it takes place in. It goes without saying that the person in this scenario has undergone taxing physical activity. In order to paint a clearer picture concerning survey campaigns, in just three days a single biologist will have walked $25 \mathrm{~km}$, away from commodities like running water or comfortable sleeping conditions, and having to work in low temperatures between 3 and $12 \mathrm{C}$.

The purpose of illustrating the work conditions for these particular campaigns is to give a graphic example on how these common occurrences in fieldwork translate over to real life examples and how this can lead any scientist to commit mistakes while working under strain. These mistakes can range from forgetting the exact mnemotechnic code for a particular transect, or even missing the first point by $15 \mathrm{~km}$ in unfamiliar terrain because they activated the wrong transect in the application to begin with.

\subsection{Development}

Persistence is a basic need for Data Collection. Initially, the data was collected using handwritten notes in templates printed for that purpose. Each sheet con- 
tains the data collected for one transect, which are then transcribed digitally to a spreadsheet

This method possesses a series of drawbacks for the scientist, given that each printed sheet consists of a grid where columns represent points in the transect (one hundred columns) and rows represent species found during the trip. While there is no fixed value of species, the number of species found is typically close to twenty five, thus approximately thirty rows are printed in the template (see Fig 1)

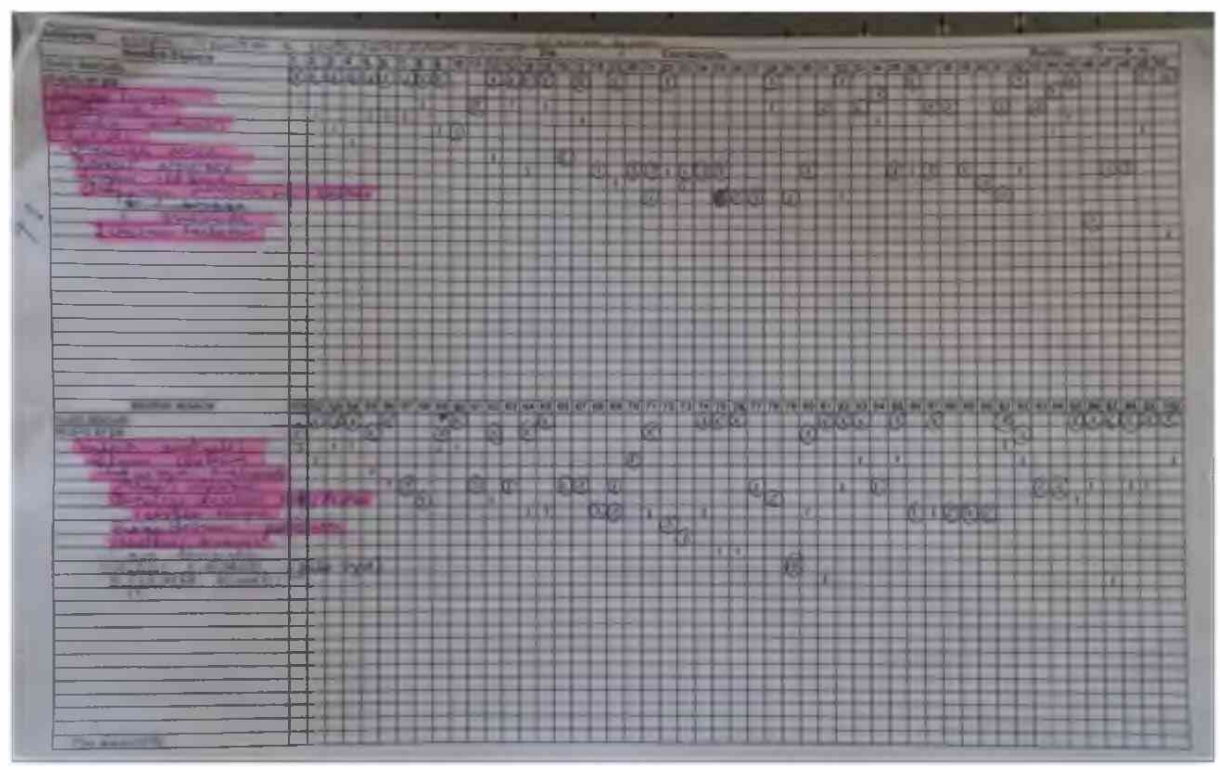

Fig. 1. Handwritten Template

The probability for human error increases as the regular long hours of fieldwork progress, and as such the opportunity for committing mistakes like misplacing data in surrounding rows or columns also grows.

The same situation can occur while transcribing the sheets, where the scientist is forced to maintain high levels of concentration in order to avoid making any possible mistakes, which can produce a significant amount of physical and mental stress.

Moreover, as each cell has a very reduced area available, if more space is required to record more information for any given data collection point, it would have to be recorded elsewhere (like a second sheet), with a specific reference to the data collection point it belongs to

In order to provide support in this task, the applications have been developed to make use of a computing area known as Context-Awareness, which in turn 
enables the change to go not just from a paper form to a spreadsheet, but rather a fully functional tool that can assist the data entry process completely.

A Context-Aware System is defined in [9] as a system that [...] adapts according to the location of use, the collection of nearby people, hosts, and accessible devices, as well as to changes to such things over time.

In the same way, Awareness and Context are defined by [10] as the use of context to provide task-relevant information and/or services to a user and define context as any information that can be used to characterize the situation of an entity, where an entity can be a person, place, or physical or computational object respectively.

Based on these definitions, different categorizations of Awareness can be applied. Table 1 describes the type of awareness implemented for the applications developed in this study.

Table 1. Implemented Awareness in each application.

\begin{tabular}{|c|c|c|}
\hline Sensibility & Sensing Method & Application \\
\hline Server Detection & Wi-Fi & LeafLab \\
\hline $\begin{array}{l}\text { Mobile Application } \\
\text { Detection }\end{array}$ & Wi-Fi & LeafLab Wear \\
\hline Adaptive Behavior & $\begin{array}{l}\text { Continual survey of } \\
\text { the species found }\end{array}$ & $\begin{array}{l}\text { LeafLab and } \\
\text { LeafLab Wear. }\end{array}$ \\
\hline Smart Suggest & $\begin{array}{l}\text { Analysis of the same } \\
\text { transect in previous } \\
\text { visits. }\end{array}$ & LeafLab Wear. \\
\hline Screen related & Based on the & LeafLab. \\
\hline Information (Help) & $\begin{array}{l}\text { user in app } \\
\text { section location }\end{array}$ & \\
\hline Location Sensitivity & GPS Sensor. & $\begin{array}{l}\text { LeafLab and } \\
\text { LeafLab Wear. }\end{array}$ \\
\hline Orientation Sensitivity & $\begin{array}{l}\text { Compass or } \\
\text { Geomagnetic Sensor }\end{array}$ & LeafLab. \\
\hline $\begin{array}{l}\text { Hand Gesture's } \\
\text { Sensitivity }\end{array}$ & Accelerometer. & LeafLab Wear. \\
\hline Energy Level & Internal System & LeafLab and \\
\hline Sensitivity & Sensor & LeafLab Wear. \\
\hline
\end{tabular}

In order to arrive at a viable solution, an application was developed (LeafLab [11]) centered around data gathering and persistence phases, and was divided in to two modules: LeafLab Mobile and LeafLab Server.

Based on the results yielded by the first version of this application, a second version (LeafLab Wear, see [12]) was elaborated with more context-awareness, as well as the possibility of adding a new device (a Smart-watch), which greatly improved the efficiency of the system, and the user's experience. Both of these versions are briefly detailed below. 


\section{$2.2 \quad$ Leaflab}

LeafLab Mobile is a hybrid mobile application (see [13-15]) developed to run in both Android and iOS based devices. The application was designed to be used in tablets, with the purpose of bringing the user all the relevant information without the need to navigate through numerous amounts of screens.

Context-Awareness was the cornerstone of this project, which meant making the application responsive to a specific set of conditions, such as the entirety of the transect, if the location corresponded to the data to be collected, device's details such as battery consumption and so on.

The application is composed by four general views (see Fig:2). The first view depicts all the general data about the actual transect (amount of data already collected, remaining transect length from navigation point, direction, and fast access to typical tasks that can be done during this phase (to add an point of interest or note, take a picture of the place and attach it to the transect, etc.).
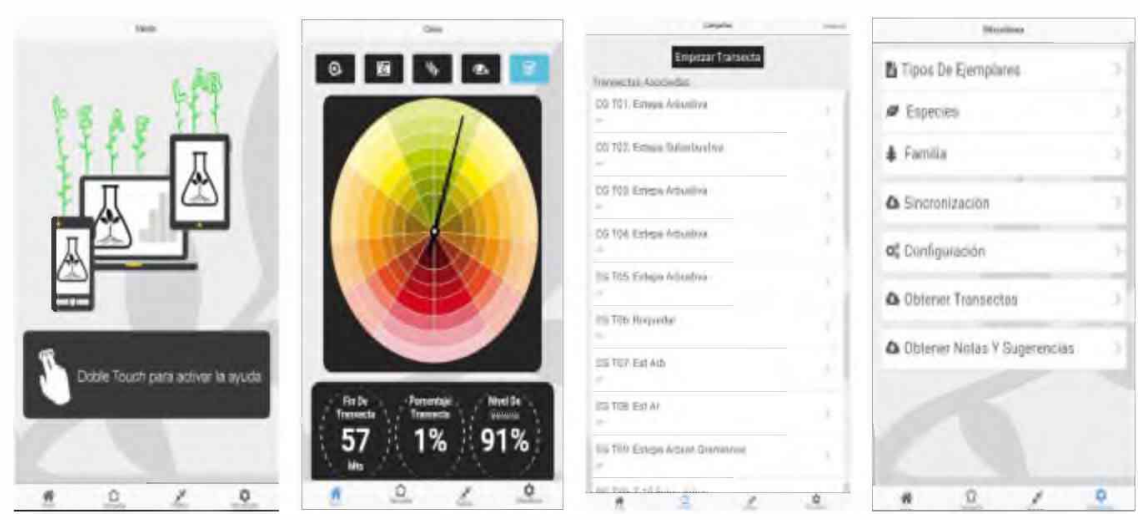

Fig. 2. Principal views of LeafLab. From left to right: Initial view (no transects activated), General information and navigation of a transect, List of collection campaigns and Miscellaneous.

In the next three views, the user can review the data that was collected in previous campaigns (collection of transects), the geolocation points in the actual transect, and to configure the set of relevant elements to be added in each point/transect (in addition to flora information).

The first step (if the application already has the information about the species to be collected), is to create and/or activate a transect. The shortcuts for the previously mentioned tasks will be made available from the principal view solely from this moment on.

To create a transect, the user can start it (create the first point) wherever they consider best, and the application will record that exact position to assist the user in future trips. 
If the user activate a previously created one (which means, is a new visit over an already existent transect), the application will guide the user to the initial point, and will antomatically activate the Information Load view when the user reach it.

The application will automatically maintain an up-to-date count of the completed points, so that the probability of inputting crroncous information between adjacent points is greatly reduced.

If the user needs to add more information it can be done through the same action menu by attaching it to the point or transect (depending on what user requires), without the need for any additional tools (except for the collection of physical samples), thus allowing a reduction in the time needed to accomplish these kinds of tasks when compared to the traditional methodology that uses physical template sheets.

In the other hand, LeafLab Server is a standalone application (the principal view of a transect can be seen in Fig 3), developed to synchronize with the mobile pair and allows the user to llave all the data that was collected in the campaigns to be backed up into a database for that purpose.

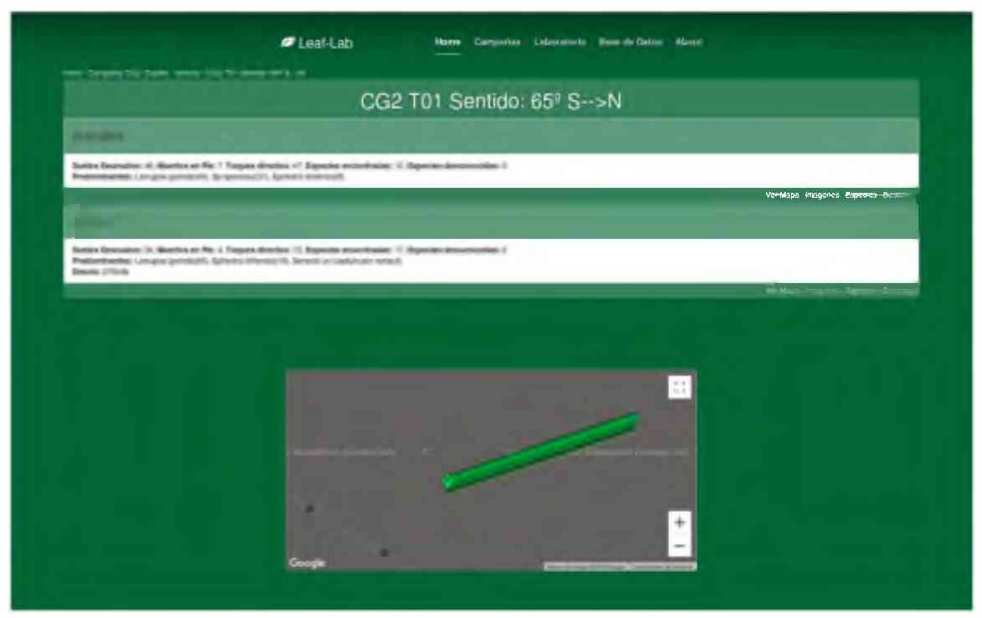

Fig. 3. Principal view of LeafLab Server for a transect.

In addition to the data persistence, LeafLab Server can visualize the information stored in the database distributed in a map according to their corresponding real position (sce Fig 4), and acess detailed information related to it, which allows the user to query for species related items, and also gives them the possibility of downloading all the information in the template-based data sheets format that they are more familiar with.

The map makes use of the Google Map API (Application Programming Interface), and was implemented with the sole purpose of providing support for the user in the selection of the transect or campaign being revised, through an easy 
to read spatial reference that could recognizes when regions had been previously visited or not. While application's map function does make use of the Google Map API, it does not provide any type of integration with GIS (Gcographic Information System) tools at this stage.

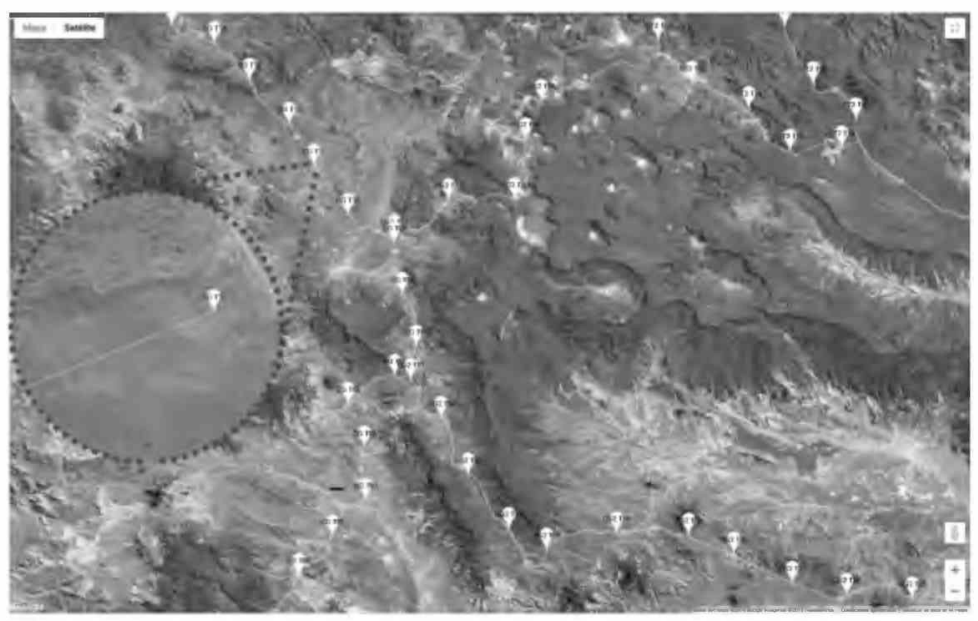

Fig. 4. View of initial points of some transects on the map.

The synchronization function is not just intended to free the user of several hours of digitization, but also to reduce the possibility of human error (caused by distraction, tiredness or stress) during the task. The synchronization was implemented using the two-phase commit protocol, in which a coordinator (in this case, the mobile app) initiates the synchronization process, while the rest of the participants (the LeafLab Server) are responsible for the transaction and informing the coordinator whether or not the request was successfully completed (by adding the data sent by the mobile application). The coordinator will abort the transaction if the server sends an Error Message warning, or if the servers take too long to submit a response. At this point, the mobile application notifies the user if the operation succeeded or failed, where they can then choose whether to make another attempt immediately or complete the synchronization at a later time.

While it is possible to use and synchronize the data base with data collected between multiple devices, this version of the software does not support concurrent connections, which means that the uscr can currently only synchronize one device at a time.

\subsection{LeafLab Wear}

In the same way LeafLab Mobile was focused in minimizing the manual effort needed for the data to be collected, LeafLab Wear was an Android Native 
project (developed making use of specific technology for Android based devices, see $[13,14])$ centered in the user experience $([16,17])$, drastically improving the transparency and efficiency of the system.

This project was based on a research about users cases, interaction, and time consumption in the previous version of LeafLab.

With this in mind, the LeafLab Wear implementation expands the previously described system with the addition of a Smart Watch application, making use of their capabilities to minimize the time the user must spend interacting with the tools that are meant to be used as support.

LeafLab Wear relies on fast and simple user gestures ([18]) to diminish direct interaction with the mobile application, and therefore allowing the scientist to focus in their job instead of on their tools.

Furthermore, new features like communication between devices (Smart Watch and Tablet), smart suggestions based on the analysis of previously collected data from the same point, and a fast selection function that is triggered via the stimuli detected with the Smart Watch were also added.

The more frequently used functions by the scientists (such as selecting the specific species found in a point, see Fig 5) can be quickly accessed via a list shown in the hand-held Smart Watch or even with a simple gesture, so the tablet just need to be used for more complicated or hardware specific tasks (like take pictures).

Shortening the user interaction time window with the tablet device provided the convenience of a longer battery life, which was especially beneficial given that accessing electrical energy is something of an impossibility at the locations where the surveys take place. This improves the overall work environment, because it frees the user from the constant concern that the device will run out of energy before the task can be fully completed.

In order for the application to display the desired characteristics, it became necessary to implement other important aspects such as Distributed User Interfaces and coordination aspects between both devices by modifying the first version of the application, which had originally only been contemplated to work independently on its own (see $[19,20]$ ).

The interfaces were semantically distributed (as detailed in $[19,21]$ ), which means that the elements the user can interact with in both the Tablet and the SmartWatch are represented in such a way that their meanings remain the same across platforms, even if it looks different from one device to another. Some of the wearable device's views can be seen in Figure 6 .

The next section details the results obtained from different experiments, as well as a comparison between the traditional methodology, and the first version of LeafLab and LeafLab Wear.

\section{Preliminary Experiments}

The experiments detailed in this section correspond to tests made to both LeafLab versions before their use in production. 


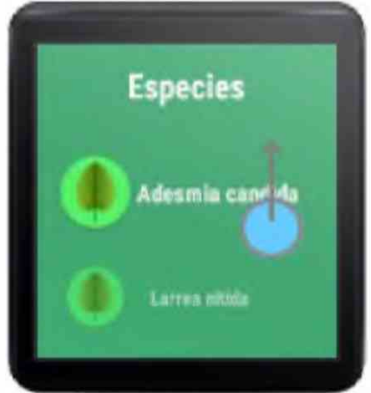

List of Species

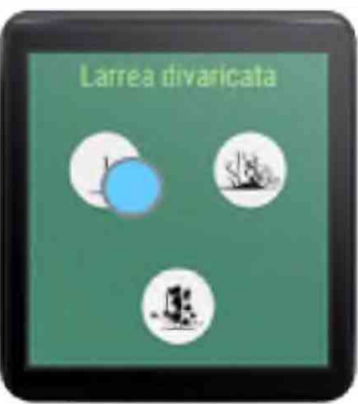

Tip type selection
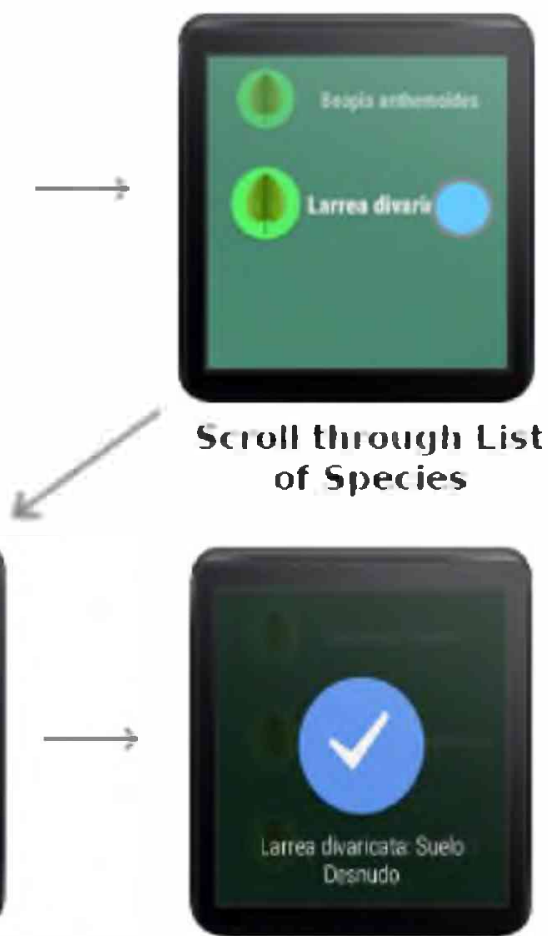

Tip) Type Selected

Fig. 5. Steps taken to complete a simple survey point.

\subsection{LeafLab}

In order to measure the performance of the mobile application in a real case, a two phase experiment was designed taking the following

- Localization: The tests were done in regions close to Telsen-Chubut (Provincial Rute 4), which presents the normal conditions for the use of the application.

- Time Span: The experimentation process lasted three days, requiring two days for the first phase, and one day for the second phase. The phases were executed in different periods of the year.

- Amount of data collected: The tests were executed using data corresponding to ten transects. Due to the aim of each phase, one thousand points were collected and analyzed for the first phase, whilst a series of measures were collected over each of the ten transect's initial points were collected for the second one.

- Test team composition: The team was composed by three developers, and a biologist from the Universidad Nacional de la Patagonia "San Juan Bosco" 's 


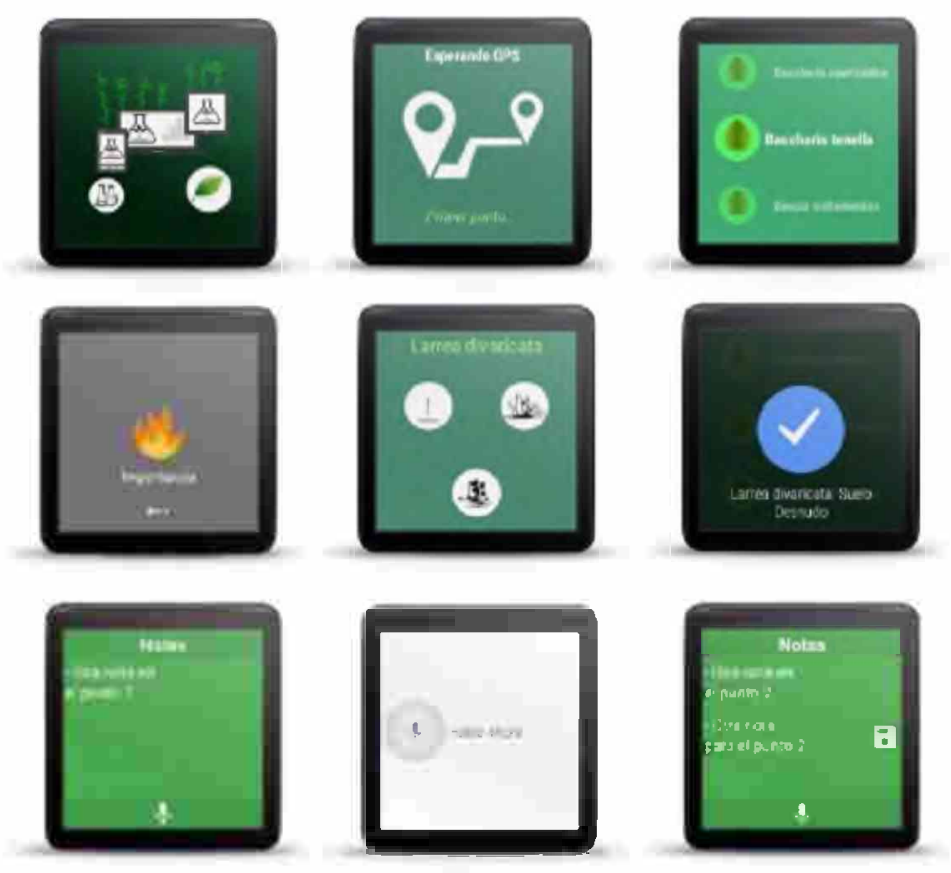

Fig. 6. Some of the views implemented for the wearable device.

Biology department, who was only familiarized with the traditional methodology, and had no background knowledge whatsoever regarding the application.

This way, the user had to learn how the application worked during the survey of the transects, and the team could collect data about the the system's learning curve.

In general terms, the measures taken correspond to the total time needed to complete a transect, average time to complete a point, fastest survey method (the traditional way or with the application), and the time required to reach the first point of a transect.

\subsection{LeafLab Wear}

In order to verify the performance of the second version of the application with the wearable component in a real time scenario, an experiment was designed taking into account the the following aspects:

- Localization: With the objective generating comparable results, the tests were performed in the same location and using the same transects as in the experimentation for the first version. 
- Time Span: The time needed for the whole experimentation phase was one day (from 5AM to 9PM), on February of 2017.

- Amount of data collected: The same transects used during the experimentation for the first version were revisited (10 transects, 1000 points).

- Test Team Composition: Again, the team was composed by three developers and a biologist, but this time the biologist was already familiarized with the first version of the application, but with no previous knowledge of the wearable application.

As in the previous case, the user had to learn how the system worked with the new device (Smart Watch) during the survey, which sped up the work flow process because of how intuitive and easy to use the device application was.

In addition to the aforementioned measurements, the number of times the mobile application was used to register a point, the number of times the smart watch was used for the same task, the number of times the smart watch could have been used, and remaining energy in both devices before and after the trip was recorded.

\section{Experimental Results}

This section depicts the results of tests performed for each version of the application. Table 2 shows the time that takes the user to reach the first point of a transect, starting from the moment the applications were ready to guide the user. At first glance, version one of the application seemed to have a better performance, however the time lost in geolocation data acquisition and delays produced by choosing a wrong transect (the user must manually choose and activate each transect) was not measured. Taking these kind of problems into account, the average time for the first version ascended to 4'42" (four minutes and forty two seconds, complete data in the Real Case Scenario section). The second version did take this into account, making the device sensitive to the actual location in a continuous way, so that the user doesn't need to wait for a GPS acquisition delay (when the vehicle stops moving, the device already has the user's geolocation). This way, the possibility of activating the wrong transect is also eliminated because the device automatically chooses the transect that is in closest proximity. This implies that in the second version, the total time necessary starts from the moment the vehicle stops until the user arrives at the first point, in contrast with the first version where the user was more likely to forfeit several minutes while the vehicle was no longer in motion or due to any of the previously explained situations.

Table 3 displays a comparison between the traditional methodology and the first version that was developed, depicting the points that were finished in the least amount of time by each method.

For the second version only the time required to complete the task using the application was recorded. Given that it was established that the use of the application itself did not delay the task, the improvement gained by using it was 
Table 2. Time it takes to reach First Point of Transect

\begin{tabular}{ccc}
\hline Transect & \multicolumn{2}{c}{ Time to First Point } \\
& LeafLab LeafLab + Wear \\
\hline 1 & $2^{\prime} 30^{\prime \prime}$ & $6^{\prime} 35^{\prime \prime}$ \\
2 & $0^{\prime} 53^{\prime \prime}$ & $1^{\prime} 50^{\prime \prime}$ \\
3 & $1^{\prime} 00^{\prime \prime}$ & $1^{\prime} 46^{\prime \prime}$ \\
4 & $1^{\prime} 12^{\prime \prime}$ & $2^{\prime} 25^{\prime \prime}$ \\
5 & $0^{\prime} 55^{\prime \prime}$ & $3^{\prime} 46^{\prime \prime}$ \\
6 & $0^{\prime} 40^{\prime \prime}$ & $4^{\prime} 26^{\prime \prime}$ \\
7 & $0^{\prime} 34^{\prime \prime}$ & $3^{\prime} 34^{\prime \prime}$ \\
8 & $1^{\prime} 23^{\prime \prime}$ & $1^{\prime} 50^{\prime \prime}$ \\
9 & $0^{\prime} 51^{\prime \prime}$ & $1^{\prime} 32^{\prime \prime}$ \\
10 & $0^{\prime} 38^{\prime \prime}$ & $1^{\prime} 43^{\prime \prime}$ \\
\hline Total & $10^{\prime} 36^{\prime \prime}$ & $29^{\prime} 27^{\prime \prime}$ \\
\hline
\end{tabular}

Table 3. Comparison between LeafLab v1 and the traditional methodology

\begin{tabular}{ccc}
\hline Transect & \multicolumn{2}{c}{ Less Time with } \\
& Traditional LeafLab (V1) \\
\hline 1 & 31 & 69 \\
2 & 35 & 65 \\
3 & 36 & 64 \\
4 & 32 & 68 \\
5 & 39 & 61 \\
6 & 33 & 67 \\
7 & 33 & 67 \\
8 & 36 & 64 \\
9 & 38 & 62 \\
10 & 26 & 74 \\
\hline Total & $33.9 \%$ & $66.1 \%$ \\
\hline
\end{tabular}

measured instead. There was approximately a $35 \%$ increase in the speed of the data collection.

\section{$5 \quad$ Real Case Scenario}

In the summers of 2016 and 2017 , the previously mentioned tools were put to use in an actual campaigns, providing the opportunity to collect a great deal of data relevant to the established parameters.

Fig 4 is a map of the geographic distribution of the 200 transects that were visited during the years 2016 and 2017, using both of the developed versions of Leaflab. The first version was used during the 2016 campaigns. During the experiment, sensitivity to context along the transect path was very limited because it had no previous records of the plant species found there due to it being the very first trip. In the course of the following year's campaigns, the second 
Table 4. Total Time to Complete a Transect

\begin{tabular}{|c|c|c|}
\hline \multirow{3}{*}{ Transed } & \multicolumn{2}{|c|}{ Total Time } \\
\hline & Leaftab & TealTab (V2) \\
\hline & + Traditional & + Wear \\
\hline 1 & $97^{\prime}$ & $39^{\prime} 25^{\prime \prime}$ \\
\hline 2 & $61^{\prime}$ & $32^{\prime} 48^{\prime \prime}$ \\
\hline 3 & $50^{\prime}$ & $32^{\prime} 04^{\prime \prime}$ \\
\hline 4 & $38^{\prime}$ & $31^{\prime} 00^{\prime \prime}$ \\
\hline $\bar{\delta}$ & $44^{\prime}$ & $28^{\prime} 17^{\prime \prime}$ \\
\hline 6 & $49^{\prime}$ & $33^{\prime} 49^{\prime \prime}$ \\
\hline 7 & $38^{\prime}$ & $25^{\prime} 12^{\prime \prime}$ \\
\hline 8 & $30^{\prime}$ & $25^{\prime} 46^{\prime \prime}$ \\
\hline 9 & $44^{\prime}$ & $38^{\prime} 20^{\prime \prime}$ \\
\hline 10 & $40^{\prime}$ & $29^{\prime} 36^{\prime \prime}$ \\
\hline Total & $491^{\prime}$ & $216^{\circ} 17^{\prime \prime}$ \\
\hline Point AVG & $45^{\prime \prime}-47^{\prime \prime}$ & $26^{\prime \prime}-30^{\prime \prime}$ \\
\hline
\end{tabular}

version of the application (in conjunction with LeafLab Wear), used the 2016 context data related to the plant species found in each transect of the campaigns in order to heighten the sersitivity to context information while the user walked along each transect line. Fig 7 depicts the progress of the tools used during the campaigns.

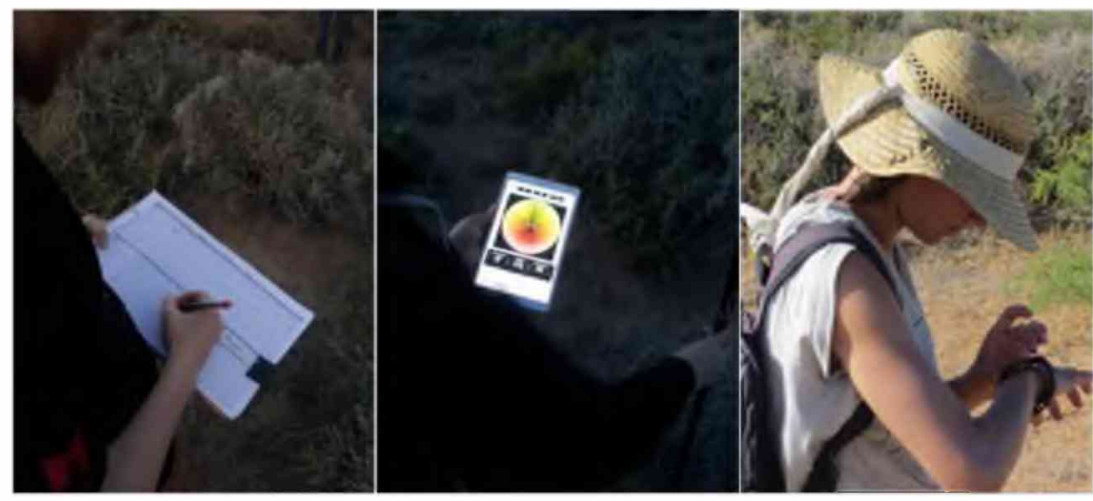

Fig. 7. From left to right: printed sheets used in traditional methodology, first version of LeafLab (Mobile), and the second version of LeafLab (Wear).

\subsection{Hypothesis Test}

Tables in [22] display the amount of time taken to complete each transect for the years 2016 and 2017, respectively. The classic statistic hypothesis t-student test 
(see [23]) was used to analyze the impact of these sample matches. Contimnous variables are being used, and large samples have been obtained (ni,30), which adjust to a normal distribution as can be observed in Fig 9.

This hypothesis test takes the following variables into account: Variable $\mathrm{x}$ : Time required to finish a transect without context sensitivity.

Variable y: Time required to finish a transect using context sensitivity.

The null hypothesis and alternate hypothesis are as follows:

H0: There are no significant differences in the observed measurements.

HA: There are significant differences in the observed measurements.

The hypothesis test results are shown in Fig 8 a. The null hypothesis was disproved due to the drastically low p-value(less than a $5 \%$ significance level), and because the sample averages differ greatly (sec Fig 8 b)..
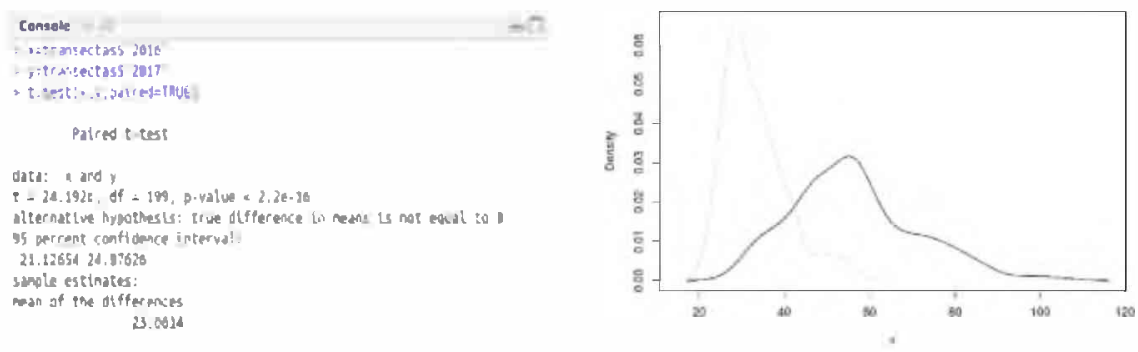

Fig. 8. From left to right: T-Test results and Density plot for minutes in 2016(black) and 2017 (gray)

When analyzing the times registered for each transect completion, it was found that after implementing the context sensitivity upgrade at least 23 minutes on average was reduced from the overall time required for the survey tasks in each transect. The Shapiro-Wilk normality test when applied to the absolute differences (Fig 9), yielded p-value $>0.05$. This allows for the difference variable (x-y) affirmation, which corresponds to a normal distribution displaying a 23.0014 minute mean and a 13.44 standard deviation (9 b).
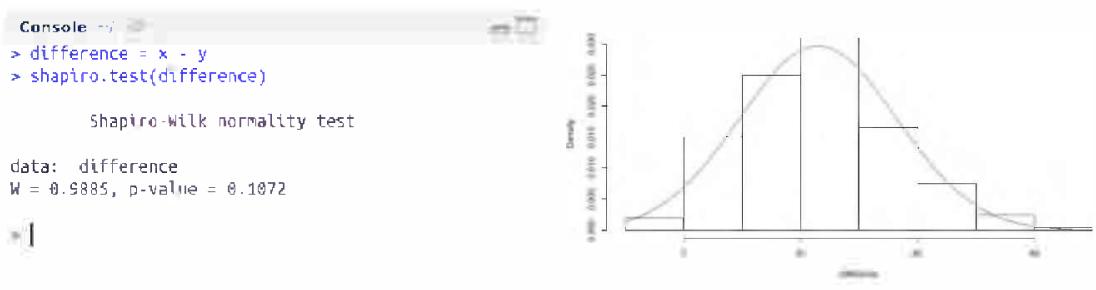

Fig. 9. From loft to right: Shapiro-Wilk tcst and Difference Histogram 
The CohenD statistic (Fig 10) for the estimation of the effect size revealed a large impact result $(\mathrm{d}>0.8)$.

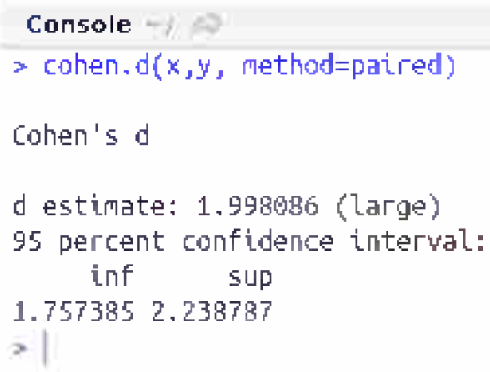

Fig. 10. Cohen'D statistic

The statistic results obtained reflect the data collection corresponding to 400 transects, where at least 100 species were registered for each transect, resulting in a 40000 species registry, aided by suggestions based on context which made their task more efficient. The total for the absolute difference between samples registered for 2016 and 2017 measures up to 76.6 less hours of work when compared to the traditional methodology.

\section{Conclusions and Future Work}

Completing the preliminary groundwork, and obtaining real life results confirmed that the use of mobile applications and wearable technology vastly improve the user's experience and efficiency in these kind of tasks, when taking into account aspects like context information, as well as the device sensors and autonomy.

During this investigation, the tools and equipment used during campaigns were reduced to only two devices: one mobile device and one wearable device. The user must charge each battery and a few portable power banks (depending on the duration of the campaign) before setting out into the field.

In exchange, the user no longer needs to prepare and print datasheets or any other resources, folders, writing utensils, cameras, compasses, and gps devices. The development of this software truly innovated the productivity and workflow, because where they had previously only been able to complete around 27 transects per season they were able to increment this number to 200 transects with the application, which implies close to a $600 \%$ increase. Additionally, extracting the information is as simple as connecting the tablet to a wifi network, and they automatically have all their data available appropriately georeferenced, with the corresponding image relations and without any fear of input errors. 
With this study we have been able to corroborate that when expanding the context sensibility in this way, not only does it provide a better experience for the user, but it also boosts the user productivity significantly.

Part of our investigation team is currently working on possible mobile applications in similar intensive data collection scenarios, where the manipulation of multiple sample objects is required for both field and lab work. A possible future goal would be to compare the data gathered in those new projects with the results exposed in this article.

Furthermore, even though all of the described software products are already in use, the possibility of including drones and image processing to improve the efficiency and labor conditions of these scientists has been contemplated for future proposals.

\section{Acknowledgments}

A special thanks to Emilio for his assistance and support, both during the experimentation periods and in the 2016 and 2017 campaigns.

\section{References}

1. David George and Tim Hatt. Global Mobile Trends 2017. https://www. gsmaintelligence.com/research/?file=3df1b7d57b1e63a0cbc3d585feb82dc2\& download, 2017. [Online; accessed 26-March-2019].

2. Peter Jarich, Tim Hatt, and David George. Global mobile trends: Whats driving the mobile industry? https://www.gsmaintelligence.com/research/?file= 8535289e1005eb248a54069d82ceb824\&download, 2018. [Online; accessed 26March-2019].

3. Sivakumaran Mayuran and Iacopino Pablo. The mobile economy 2018. https:// www.gsmaintelligence.com/research/?file=061ad2d2417d6ed1ab002da0dbc9ce 22\& download, 2018. [Online; accessed 26-March-2019].

4. Brian Whitworth, Adnan Ahmad, M Soegaard, and RF Dam. The encyclopedia of human-computer interaction, 2013.

5. Bonifacio Mostacedo and Todd Fredericksen. Manual de métodos básicos de muestreo y análisis en ecología vegetal. Proyecto de Manejo Froestal Sostenible (BOLFOR), 2000.

6. CRW Spedding and RV Large. A point-quadrat method for the description of pasture in terms of height and density. Grass and forage science, 12(4):229-234, 1957.

7. Néstor Elissalde, Juan María Escobar, and Viviana Nakamatsu. Inventario y evaluación de pastizales naturales de la zona árida y semiárida de la patagonia. Ed. PAN-SDSyPA-INTAGTZ, Trelew, Argentina, 2002.

8. Lenore Fahrig. Effects of habitat fragmentation on biodiversity. Annual review of ecology, evolution, and systematics, 34(1):487-515, 2003.

9. Bill Schilit and Marvin M. Theimer. Theimer, m.m.: Disseminating active map information to mobile hosts. ieee netw. 8(5), 22-32. Network, IEEE, 8:22-32, 10 1994. 
10. Gregory D Abowd, Anind K Dey, Peter J Brown, Nigel Davies, Mark Smith, and Pete Steggles. Towards a better understanding of context and context-awareness. In International symposium on handheld and ubiquitous computing, pages 304-307. Springer, 1999.

11. Samuel Almonacid and Pablo Navarro. Aplicaciones móviles multiplataforma sensibles al contexto: una aplicación científica para el relevamiento florístico. In $X I X$ Concurso de Trabajos Estudiantiles (EST 2016)-JAIIO 45 (Tres de Febrero, 2016)., 2016.

12. Bruno Alfredo Pazos and Arturo Leonardo Morales. Computación corporal: Expansión de la sensibilidad computacional hacia mejores experiencias de usuario. In XXI Concurso de Trabajos Estudiantiles (EST)-JAIIO 47 (CABA, 2018), 2018.

13. Tony Wasserman. Software engineering issues for mobile application development. FoSER 2010, 2010.

14. Andre Charland and Brian Leroux. Mobile application development: web vs. native. Communications of the ACM, 54(5):49-53, 2011.

15. Peixin Que, Xiao Qiao Guo, and Maokun Zhu. A comprehensive comparison between hybrid and native app paradigms. 2016 8th International Conference on Computational Intelligence and Communication Networks (CICN), pages 611-614, 2016.

16. Marc Hassenzahl and Noam Tractinsky. User experience-a research agenda. Behaviour 6 information technology, 25(2):91-97, 2006.

17. Virpi Roto. User experience from product creation perspective. Towards a UX Manifesto, 31, 2007.

18. Zhiyuan Lu, Xiang Chen, Qiang Li, Xu Zhang, and Ping Zhou. A hand gesture recognition framework and wearable gesture-based interaction prototype for mobile devices. IEEE transactions on human-machine systems, 44(2):293-299, 2014.

19. Jean Vanderdonckt et al. Distributed user interfaces: how to distribute user interface elements across users, platforms, and environments. Proc. of XI Interacción, $20,2010$.

20. Alexandre Demeure, Jean-Sébastien Sottet, Gaëlle Calvary, Joëlle Coutaz, Vincent Ganneau, and Jean Vanderdonckt. The $4 \mathrm{c}$ reference model for distributed user interfaces. In Fourth International Conference on Autonomic and Autonomous Systems (ICAS'08), pages 61-69. IEEE, 2008.

21. Niklas Elmqvist. Distributed user interfaces: State of the art. In Distributed User Interfaces, pages 1-12. Springer, 2011.

22. Diego Andrés Firmenich. Transects times. https://igshare.com/articles/ Transects_times/7862909, 2019. [Online; accessed 26-March-2019].

23. Jarrett Rosenberg. Statistical methods and measurement. In Guide to Advanced Empirical Software Engineering, pages 155-184. Springer, 2008. 